\title{
EL JUEGO DE PELOTA PREHISPÁNICO Y LOS JUEGOS OLÍMPICOS
}

\section{PREHISPANIC BALL GAME AND THE OLYMPIC GAMES}

\author{
Juan Gabriel Ocampo Hurtado ${ }^{1}$ \\ 1 PhD. en Arquitectura, Diseño y Urbanismo. Universidad Nacional de Colombia. Calle 57 No 24 A 36 Manizales, Colombia. \\ jgocampoh@unal.edu.co
}

Rev. U.D.C.A Act. \& Div. Cient. 15 (Supl. Olimpismo): 17 - 25, 2012

\section{RESUMEN}

El deporte, como componente fundamental en la formación integral de personas, encuentra en la cultura su riqueza, como instrumento de valoración al sentido de lugar en todas las regiones del mundo. El juego de pelota prehispánico, con sus más de tres mil años de existencia, merece la categoría de juego olímpico histórico, a partir de su aporte cultural. Frente a dicho objetivo, la triangulación hermenéutica, como método, permite tener una interpretación sobre la relación entre deporte y cultura. Como resultado de esta investigación, la ciudad pirámide de Xochicalco, perteneciente al período Epiclásico mesoamericano, sirve de ejemplo para entender la motivación del deportista, que se dio en ese caso y en su modalidad sagrada, en la tensión entre el inframundo y la existencia en un mundo superior.

Palabras clave: Historia latinoamericana, cultura amerindia, juegos olímpicos, sitio arqueológico.

\section{SUMMARY}

The sport like fundamental component in the integral formation of persons finds in the culture his wealth as instrument of valuation to the sense of place in all the regions of the world. The pre-Hispanic ball game, with more than three thousand years of existence, deserves the category of Olympian historical game from his cultural contribution. With this objective the hermeneutics as a method of triangulation allows an interpretation of the relationship between sport and culture. As a result of this research, the pyramid city of Xochicalco, belonging to the Mesoamerican Epiclasic period, serves as an example to understand the motivation of the athlete, who gave itself in this case and in the sacred modality, in the tension between the underworld and the existence in a higher world.
Key words: Latin American history, amerindian cultures, olympic games, archaeological sites.

\section{INTRODUCCIÓN}

La educación y el deporte en la formación integral:

La educación, tantas veces maltratada, menospreciada y desatendida en Colombia, es la que permite construir estrategias para una efectiva construcción de país y para la transformación real de personas (Ruiz, 2010). Las diferencias en términos de estructuración mental entre Naciones, como Colombia y Suiza, se derivan de factores inherentes a los procesos educativos, que tienen entre sí; la disciplina es ejemplo de ello. Mientras el ciudadano suizo usualmente la posee como parte de su forma de ser, el colombiano, dentro de su idiosincrasia, se aleja, en muchos casos, de este valor. Ahora bien, si la indisciplina formara parte de la identidad de una comunidad o población sería necesario afirmar que la falta de rigor también entraría a formar parte de los elementos constitutivos que identifican a dicha comunidad. Ese tipo de lógica puede resultar como una provocación frente a la calidad del sistema educativo nacional y, de hecho, bien vale la pena motivar la discusión y la reflexión sobre el tema, ya que las universidades, los colegios, las escuelas y las demás entidades e institutos dedicados a la enseñanza, tienen el compromiso de responder a las necesidades reales del país.

El aletargamiento presente en este sector ha sido combatido, en ciertas ocasiones, por pensadores, como Rodolfo Llinás y Gabriel García Márquez, quienes lideraron en los años 90, el comité de los "diez sabios"; sin embargo, la lucha sobre la construcción de país debe involucrar no solo a los "diez sabios" sino permear de forma consciente a los diferentes sectores involucrados, con el desarrollo nacional. 
La crisis en el sector educativo afecta de forma directa la estructuración mental de los estudiantes que en el futuro serán los líderes y los formadores de las siguientes generaciones. La dificultad en las búsquedas y en la construcción de estrategias educativas fomenta un fenómeno de inercia estática, en la cual, un país con la enorme riqueza en recursos de toda índole, como lo es Colombia, llegue a los problemas sociales existentes.

Pierre de Coubertin, educador francés, consciente del "agotamiento escolar", fundó en 1906, la Asociación para la Reforma de la enseñanza pública, a partir, de la cual, dedicó buena parte de su vida a la investigación y a la construcción de estrategias para un mejor e integral proceso formativo, que incluiría a la postre la educación de la inteligencia y del cuerpo.

Coubertin creó los juegos olímpicos modernos (Nielsen, 1977), como estrategia para recuperar los ideales clásicos deportivos, con tal éxito que se llegó a convertir en un referente, como proyecto de unidad global contemporánea, superando fronteras y diferencias políticas.

Los ideales clásicos que perseguía Coubertin, se encontraban en los juegos olímpicos de la antigüedad, que se realizaban en Olimpia, en honor a los dioses. El primer referente histórico, se refiere a un escrito de Hipias de Elis, que data de 776 A.C., en el cual, además de mencionar que la primera carrera de atletismo fue la prueba de 200 metros, elaboró un listado con los vencedores del certamen, realizado en aquella época (Briceño, 1990).

\section{Los Juegos Olímpicos en Latinoamérica:}

Los Juegos Olímpicos modernos han tenido como sede diferentes países, desde que se realizaron por primera vez en Atenas, en 1896. El propósito de llevar la sede a un nuevo país en cada ocasión que se realice, responde al sentido original de unir a la humanidad por la fuerza del deporte. Coubertin logró llevar su intención pedagógica de una educación integral a todos los países del mundo.

Actualmente, existe la posibilidad, de acuerdo con el Comité Olímpico Internacional, que Colombia sea sede de este certamen. Este hecho tiene un significado profundo, puesto que México, en 1968, fue el primer y único país latinoamericano que ha tenido este honor, en la historia de los Juegos Olímpicos. En dicha ocasión, se dio un evento trágico con la muerte de más de 80 personas, luego de una manifestación estudiantil, realizada una semana antes de la inauguración. En ese caso, el sentido del deporte, como componente estructural pedagógico, perdió fuerza frente a las dificultades políticas inherentes a las discusiones sobre la inversión de recursos públicos.
El sentido original de los Juegos Olímpicos responde a una búsqueda por mejores formas pedagógicas, que incluyan la formación integral como objetivo. El conocimiento y el deporte pueden formar parte de esta estrategia. La cultura, en todas sus manifestaciones, tiene relación directa con dicho carácter integral, puesto que la praxis de cada individuo se inicia en su contacto particular con el mundo. Esto significa que todos los lugares, en su sentido profundo, afectan el conocimiento y la experiencia de las personas.

Latinoamérica, como región, ofrece su enorme diversidad multicultural, como aporte a la celebración de los juegos olímpicos. Para Colombia, la oportunidad presente puede establecer un contexto de discusión que posibilite la construcción de conocimiento frente a las reformas educativas nacionales.

Entre los temas de reflexión posibles, se encuentra la inclusión de deportes en la categoría de olímpicos. Si bien existen deportes olímpicos que no se encuentran en la programación de los Juegos Olímpicos, valdría la pena investigar en los deportes latinoamericanos tradicionales, sobre la posibilidad de su inclusión en esta categoría.

\section{El Juego de pelota:}

En la ciudad prehispánica mesoamericana, se encuentran múltiples elementos arquitectónicos que las hacen partícipes de un conjunto de culturas provenientes de la misma raíz. Entre los elementos presentes, en la generalidad de las ciudades arqueológicas, se hallan la pirámide, la plaza y el juego de pelota. La pirámide, por su parte, señala al cielo y se ubica en lo alto de las ciudades; la plaza, a continuación de la pirámide, establece la relación entre el pueblo y el mundo superior y, por su parte, el juego de pelota, se localiza en los sectores bajos, para entrar en relación con el inframundo. De esa forma, la plaza se presenta como elemento articulador entre ambos mundos.

Si bien en lo profundo y a partir de esta lógica se encuentra el inframundo, también es cierto que la muerte da paso a la vida y es así como desde lo profundo de las cañadas nace el agua que da paso a la vida. Esta lucha entre la vida y la muerte se escenifica en el juego de pelota, en la forma de ritual de fertilidad, que se lleva a cabo en honor al dios Huitznáhuatl, en el mes de panquetzaliztli (Martínez, 2008).

Sin embargo, no solo lo sagrado tenía cabida en el juego de pelota; el carácter profano también tenía su espacio (Sahagún, 1829), a modo de juego de azar o en términos de autorización a un enemigo capturado, para morir con honor en el juego (Schele \& Miller, 1986). Esta modalidad de juego de pelota se realizaba en cualquier momento del año, con objetivos lúdicos específico. 
La estrecha, aunque lejana relación en el espacio-tiempo entre los juegos olímpicos y la práctica del juego de pelota prehispánico mesoamericano, enfatiza la fuerte relación existente entre el deporte y la cultura. Esta es una forma de evidenciar la relación entre naturaleza y cultura que señala la corriente de pensamiento ambiental latinoamericano (Noguera, 2004), como una ruta para proteger al planeta de los abusos cometidos por el ser humano. Los Juegos olímpicos, como forma de abordar la búsqueda de soluciones a problemas de orden global, pueden llevar a la posibilidad reflexiva sobre la importancia de la cultura, en el sentido de la existencia humana. El juego de pelota prehispánico mesoamericano es un ejemplo de actividad deportiva, que acompañaba el significado de un pueblo.

La investigación "Lógica y Arquitectura", se plantea como ruta de búsqueda sobre el sentido del lugar. Para ello, se profundiza en las relaciones existentes entre naturaleza y cultura en sitios y situaciones que definen circunstancias de lugar. La presencia posible de los Juegos Olímpicos en Colombia, aunada a la evolución sobre la investigación de Xochicalco y más precisamente al juego de pelota, plantean a Latinoamérica como macro región, en la cual, la variedad del sentido de lugar expresa la idiosincrasia de estos pueblos. Las historias que se aprecian en Latinoamérica son ricas en discontinuidades y, es por ello, que la búsqueda de relaciones entre temas, problemas y lugares abre puertas a la construcción de nuevo conocimiento. A partir del objetivo fundamental de la investigación, que se refiere a la aplicación rigurosa de la lógica en la investigación arquitectónica basada en el uso de la percepción, se elige por su riqueza cultural, al juego de pelota prehispánico, como escenario y actividad deportiva, rica en valores históricos americanos. Al plantear, como objetivo concreto la reivindicación del juego mencionado en la categoría de deporte histórico, se atiende a la riqueza en el sentido de lugar en este continente. Se propende, así, por el fortalecimiento de la conciencia del valor que el deporte tiene en la formación de personas.

\section{MATERIALES Y MÉTODOS}

El material de esta investigación ha sido obtenido de manera directa y en mayor medida en la región conocida como Mesoamérica y con más exactitud en los estados de Morelos, de México, de Yucatán, de Tabasco y de Chiapas, donde se obtuvo información directa de sitios, como Xochicalco, Teotihuacán, Chichén Itza, La Venta y Palenque, entre 2006 y 2009. Por otra parte, el apoyo de los arqueólogos mexicanos Norberto González Crespo y Silvia Garza Tarazona ha sido fundamental. A partir de dicha información, la investigación sobre el sentido del lugar ha tenido importantes resultados, como el fortalecimiento del Grupo de Trabajo Académico de Medios de Expresión y Comunicación de la Universidad Nacional de Colombia, en la
Sede Manizales, la ampliación de la red académica, mediante la articulación de este grupo, con un número significativo de universidades mexicanas que se reúnen alrededor del Doctorado en Arquitectura, Diseño y Urbanismo y la creación de la línea de investigación en poética y arquitectura. De esta forma, la relación entre pregrado y postgrado, se propone como un escenario donde la investigación y la docencia se encuentran en un mismo escenario.

\section{RESULTADOS Y DISCUSIÓN}

Entre la rica actividad deportiva latinoamericana hay una de singular importancia, en términos de cultura. Se denomina "juego de pelota" (Fotografía 1A) y tiene mayor antigüedad a los antiguos juegos olímpicos de Olimpia. Se originó, aproximadamente, en el siglo XV A.C. (Martínez, 2008) y aunque se dejaron de practicar con las reglas originales en el siglo XV D.C., existe evidencia de su práctica, bajo una modalidad contemporánea, en el sur de México.

El juego de pelota prehispánico y los Juegos Olímpicos: El juego de pelota procede de la zona conocida como Mesoamérica, que comprende parte de México, de Guatemala, de El Salvador, de Belice, de Honduras, de Nicaragua y de Costa Rica. Estos países tienen en común el origen de su cosmovisión, además de un sinnúmero de rasgos culturales, que les define como región.

Entre los documentos que enriquecen la cultura de esta región, se encuentra el Popol Vuh o libro sagrado de los mayas (Anónimo, 2003), donde los Kiche de Chichicastenango, en Guatemala, recopilaron leyendas hasta hacerse públicas, en 1701. Entre otros temas, incluida la creación del mundo, el Popol Vuh se refiere a dos gemelos divinos, conocidos como Hunahpu y Xbalanceh, quienes fueron excelentes jugadores del juego de pelota, al punto de vencer a los señores de $\mathrm{Xi}$ balbá, el inframundo en la mitología maya. De esta forma, se presenta una fuerte relación entre el juego de pelota y la lucha entre el inframundo o la muerte y el mundo de la vida (Fernández et al. 2006); no obstante, se ha encontrado evidencia que este juego servía como escenario de ritual divino, pero también para encuentros de orden terrenal y meramente humano. Otras fuentes que documentan la existencia del juego de pelota y sus características son los códices náhuatl y mayas, y las narraciones de cronistas españoles, procedentes de las épocas de conquista y de colonia.

La cancha, constituida normalmente por dos estructuras paralelas e inclinadas, paramentan un espacio horizontal central y longitudinal, que remata sus extremos por espacios transversales, para dar la forma de doble $\mathrm{T}$, que le caracteriza. Si bien las canchas del juego de pelota tienen variaciones a su forma (Martínez, 2008) existen elementos que los identi- 
fican como una misma unidad arquitectónica, destinada a la actividad sacra o deportiva. El objetivo fundamental de este juego era pasar de un lado al otro una pelota de caucho macizo sin emplear manos ni pies para impactar los marcadores en las paredes, que definían el espacio de la cancha.

Fray Bernardino de Sahagún (1499 - 1590) describió, a partir de las narraciones obtenidas por parte de los indígenas, el ritual que acompañaba al juego de pelota, en su libro Historia General de las cosas de la Nueva España; a partir de su narración, se puede hacer la siguiente síntesis:

Durante el segundo día del mes de panquetzaliztli se da inicio a la danza denominada areito, que va acompañada por cantos a Huitzilopochtli. Estas fiestas duraban veinte días y se llevaban a cabo entre horas de la tarde y las diez de la noche; luego de este inicio, se procedía, en acompañamiento de música con el sacrificio, por desmembramiento en muchos casos, de cuatro esclavos en el Teotlachtli, que es como se denominaba al patio del juego de pelota. Se finalizaba el ritual con bailes, cantos, comida y bebida, para dar paso al inicio del juego sobre la cancha que se encontraba para ese momento, embadurnada de sangre.

Al poner en contraste el juego de pelota mesoamericano con los juegos olímpicos originales, se percibe que la mayor diferencia es la de su origen geográfico, puesto que se aprecia coincidencia en algunas de las prácticas más significativas. En Grecia, por ejemplo, antes de iniciar las competencias, los competidores sacrificaban un cerdo en honor a Zeus. En Mesoamérica, por su parte, el sacrificio humano o animal, era inherente a la competencia; dicho sacrificio no se realiza en el presente ni en los juegos olímpicos ni en las versiones actuales del juego de pelota y valdría la pena profundizar en las investigaciones sobre este juego para reivindicarle, como aporte al deporte, en un sector significativo de Latinoamérica, como es Mesoamérica.

La cancha del juego de pelota se denomina, en lengua náhuatl, tlachco y la práctica del juego tlachtli (Uriarte Catañeda, 1991). Las superficies de la cancha, al igual que la generalidad de la arquitectura prehispánica mesoamericana, estaban construidas en roca, recubiertas posteriormente con cal y pintadas con minerales de colores vivos. En el piso, una franja negra o verde dividía el tlachco en dos sectores; en cada uno de estos sectores y en las paredes que delimitan el espacio, se ubicaban los anillos, denominados tlachtemalácatl, que eran los empleados como objetivo para la pelota de hule. Los jugadores vestían cinturón de cuero, casco, mandil, rodilleras, guantes y protección para los brazos.

De los seis jueces requeridos para este juego, el principal era el Huey Tlatoani o gobernante de la ciudad. Estos jueces llevaban la cuenta del puntaje; para tal efecto se contaba con las siguientes reglas (Taladoire, 2000):

- $\quad$ El único momento en el que se puede tocar con la mano la pelota era en el saque.

- No se puede tocar la pelota con la mano, con el pie o con la cabeza.

- Se puede golpear la pelota con antebrazos, hombros, espalda y nalgas.

- Faltas: al tocar la pelota con partes del cuerpo no autorizadas, al arrojar la pelota fuera de la cancha y al tener una mala recepción de pelota.

- Para ganar el juego hay dos alternativas: pasar la pelota por cualquiera de los dos aros marcadores u obtener puntaje.

- Los equipos estaban integrados normalmente por siete jugadores, aunque este número podía variar.

La presión deportiva: El calmécac era el sitio donde se daba formación a los jóvenes nobles. Allí, se daba instrucción sobre el juego de pelota y su práctica sacra. La clase social elevada, a la cual, pertenecían nobles y sacerdotes, se denominaba pipiltin; sin embargo, hombres libres pertenecientes a la clase macehualtin podían participar del calmécac, cuando tenían condiciones físicas sobresalientes. Por otra parte, existía otro tipo de jugador, que era el que participaba en los juegos de pelota profanos.

La exigencia deportiva del juego de pelota mesoamericano era muy elevada. Los jugadores en su preparación previa al juego tenían el siguiente rito:

1. Ayuno a modo de purificación

2. Contacto con el Huey Tlatoani

3. Expulsión de los malos espíritus presente en los fieles

4. Sahumerio con copal

5. Oración

6. Sacrificio animal o humano

En Xochicalco, Morelos, existe una amplia zona de preparación para los jugadores del juego de pelota (Fotografía 1B). Entre las instalaciones que allí se aprecian, se encuentra un espacio amplio y cubierto, a modo de estancia de descanso, una estructura con las condiciones de funcionamiento de un pequeño estanque o piscina y un área abierta, previa al sendero de aproximación al tlachco.

Al finalizar el juego, el capitán ganador podía ser ofrendado a los dioses (Martínez, 2007); muchas cosas dependían del juego de pelota: el honor, las propiedades, la fertilidad y supervivencia de una ciudad o simplemente el entretenimiento de la nobleza y del pueblo. Seguramente, el juego de pelota prehispánico despertó las pasiones similares a las que se han vivido una y otra vez en los juegos olímpicos. 


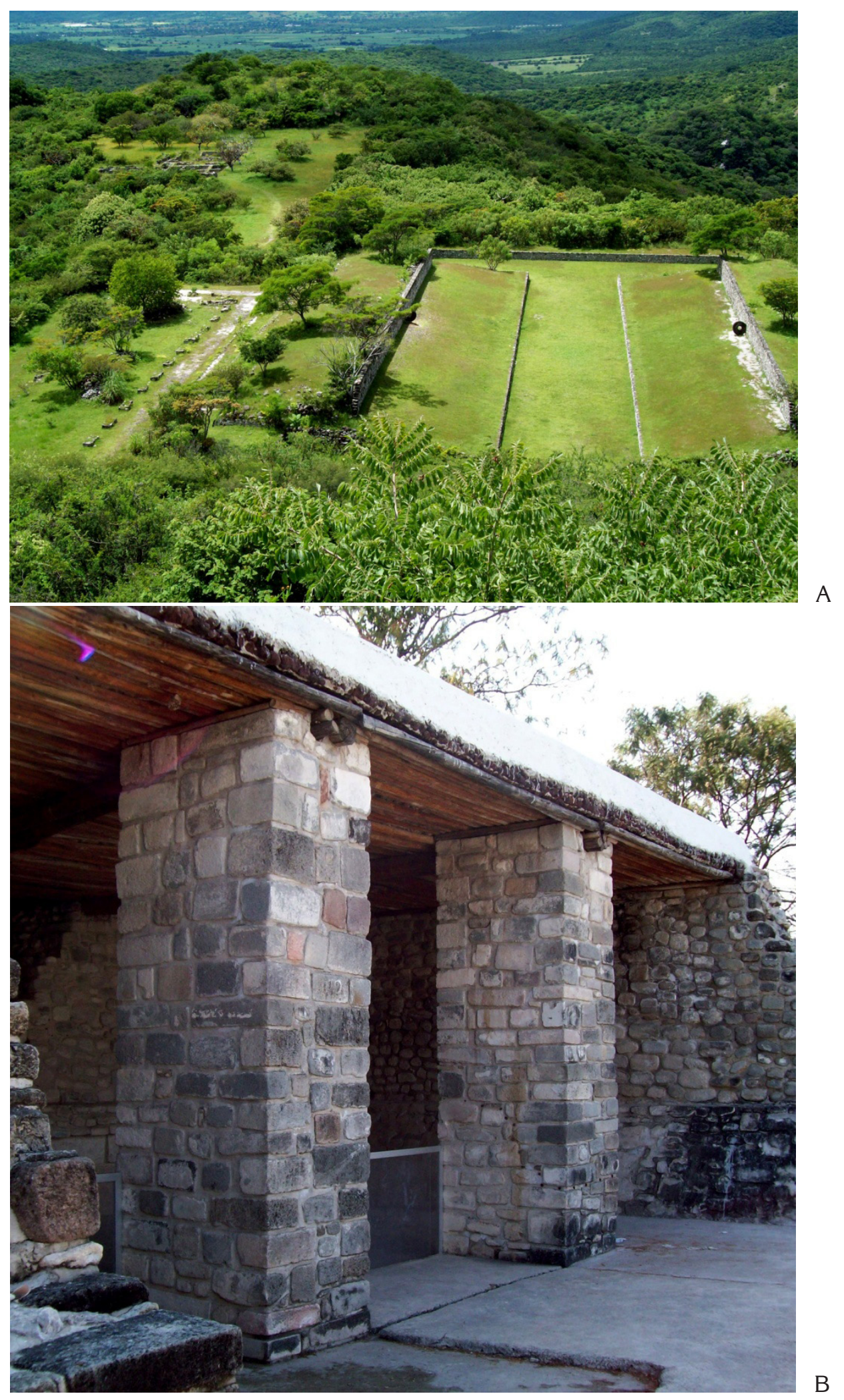

Fotografía 1. A) Juego de Pelota prehispánico. Xochicalco. B) Estructura de preparación para Juego de Pelota. Xochicalco. Fotos: Juan Gabriel Ocampo. 
La pregunta sobre la pertinencia de la relación entre el juego de pelota prehispánico mesoamericano y los Juegos Olímpicos, se refiere al diálogo entre la cultura de un pueblo y la importancia del deporte, como parte de la formación de personas. Dicha relación, se pone de manifiesto y se llega a la propuesta de categoría de juego olímpico histórico.

La propuesta de dicha categoría, se hace para efectos de incentivar la búsqueda de formas pedagógicas integrales en reacción a situaciones críticas de sistemas educativos, existentes en la actualidad.

El deporte posee sentidos trascendentales, como el de colaborar en la estructuración mental de las personas. Esa es razón suficiente para que las Universidades fortalezcan no solo sus escenarios deportivos sino sus propuestas pedagógicas, en búsqueda de la formación integral. Sobre la pregunta de cómo hacer para que la relación entre el juego de pelota prehispánico y los juegos olímpicos aporten a la reflexión sobre la formación integral de estudiantes y, en general, de personas, se propone la reivindicación de valores culturales tradicionales, que se han perdido a lo largo del tiempo. La importancia de la historia es trascendental y permite, a partir de la consciencia, construir caminos para labrar un mejor futuro. Un ejemplo de ello es la formación integral que se deriva de la relación entre el calmécac y el juego de pelota. El conocimiento sobre el pasado revela, una y otra vez, su valor en la construcción de rutas evolutivas, en ámbitos como el educativo y el científico.

El método de la triangulación hermenéutica: La necesidad de hacer un reconocimiento al juego de pelota, como deporte susceptible de ser categorizado como juego olímpico, a partir de su preponderancia histórica en Mesoamérica, surge a partir de la investigación denominada "Lógica y Arquitectura", que se adelanta actualmente en el Grupo de Trabajo Académico de Medios de Expresión y Comunicación, de la Universidad Nacional de Colombia, en la Sede Manizales. A partir de estas investigaciones, el sentido de lugar en la arquitectura, en los escenarios arquitectónicos y esto incluye los deportivos o en las actividades humanas, como parte de los hechos que definen el sentido de lugar, se encuentra que el sentido de los juegos olímpicos tiene una estrecha relación con ámbito cultural, que afecta la estructura de formación en las propuestas didácticas nacionales. Colombia forma parte de la región latinoamericana y, como tal, existen excelentes diversidad de deportes antiguos o modernos con fuerte raíz cultural. En este país, por ejemplo, se registra el tejo o turmequé, como deporte nacional, con una existencia aproximada de 500 años.

La triangulación hermenéutica, como método, facilita la interpretación a las relaciones entre naturaleza y cultura, que se dan entre el ámbito deportivo, el educativo y el arquitectónico. La adquisición de información proveniente de la razón, se pone en tensión con las ideas provenientes de la percepción sensorial profunda en los juegos de pelota de diversas ciudades arqueológicas de Mesoamérica, para tener ejecución de labores de investigación en la ciudad arqueológica de Xochicalco, en Morelos, México. Allí, tres escenarios de juego de pelota presentan contraste entre sí para responder a las condiciones de esta ciudad pirámide, del período Epiclásico, 600 a 1000 D.C. (Ocampo, 2007).

La experiencia sensorial vivida en los diferentes escenarios de Xochicalco aporta un volumen considerable de datos perceptivos que, al entrar en el ejercicio de la hermenéutica, perciben la tensión con la información procedente de otras fuentes de conocimiento, en relación con el mismo objeto de estudio. Dicha tensión lleva a la construcción de nuevo conocimiento y la estructuración de nuevas rutas de investigación.

El calmécac, en Xochicalco, era fundamental y altamente especializado, dada la importancia que tenía esta ciudad en el saber de la época. La astronomía, la técnica constructiva, la acústica, la política, la religión y la milicia, se cuentan entre los campos del conocimiento, que se dominaban en esta ciudad. De hecho, en una de sus estructuras principales, la Pirámide de las Serpientes Emplumadas (Fotografía 2A) existe evidencia en alto relieve (Fotografía 2B), sobre una reunión de carácter "internacional", del nivel de lo que sería equivalente a los juegos olímpicos

Las tres zonas de juego en Xochicalco, se hallan orientadas con respecto a la trayectoria solar. El del sur y el del norte cuentan con una orientación oriente-occidente, mientras que el Juego de Pelota oriente (Fotografía 3A) tiene una orientación norte-sur, con la cual, encuentra alineación entre el acceso principal a la ciudad y el eje que separa la zona de la ciudad, orientada a la mañana y la orientada a la tarde. Este eje conduce del inframundo al cielo.

En los juegos sur y norte, se dan connotaciones físicas intensas con respecto a su uso. El Juego de Pelota Sur (Fotografía 3B) es el más grande de los tres y se localiza más bajo que los otros dos. Los rasgos geográficos que lo circundan posibilitan la presencia masiva de habitantes. Caso contrario ocurre con el Juego de Pelota Norte (Fotografía 4A), que se ubica en lo más recóndito de la elevada Acrópolis, donde el dramatismo del sitio, lo privado del mismo y las instalación de preparación de los jugadores, previo a la celebración sagrada, se refieren a un tipo de juego, donde la vida y la muerte podrían haber llegado a la confrontación y al sacrificio (Fotografía 4B). 


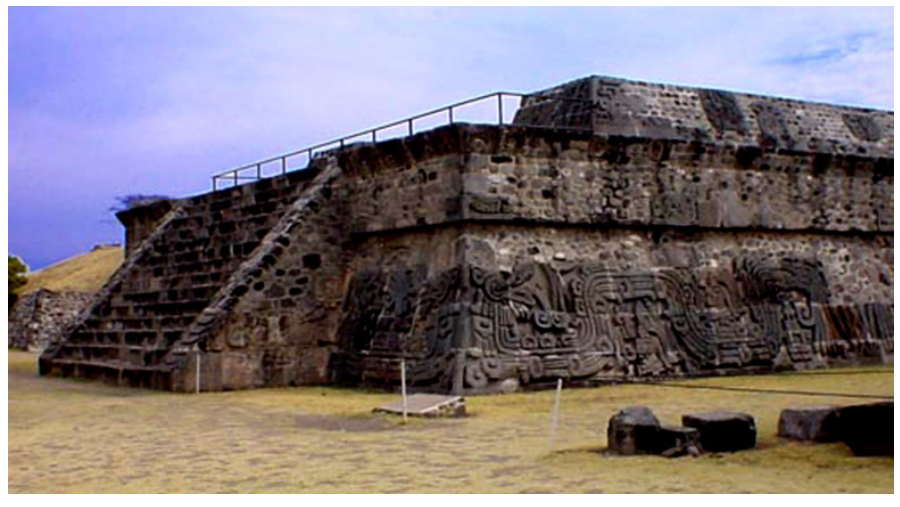

A

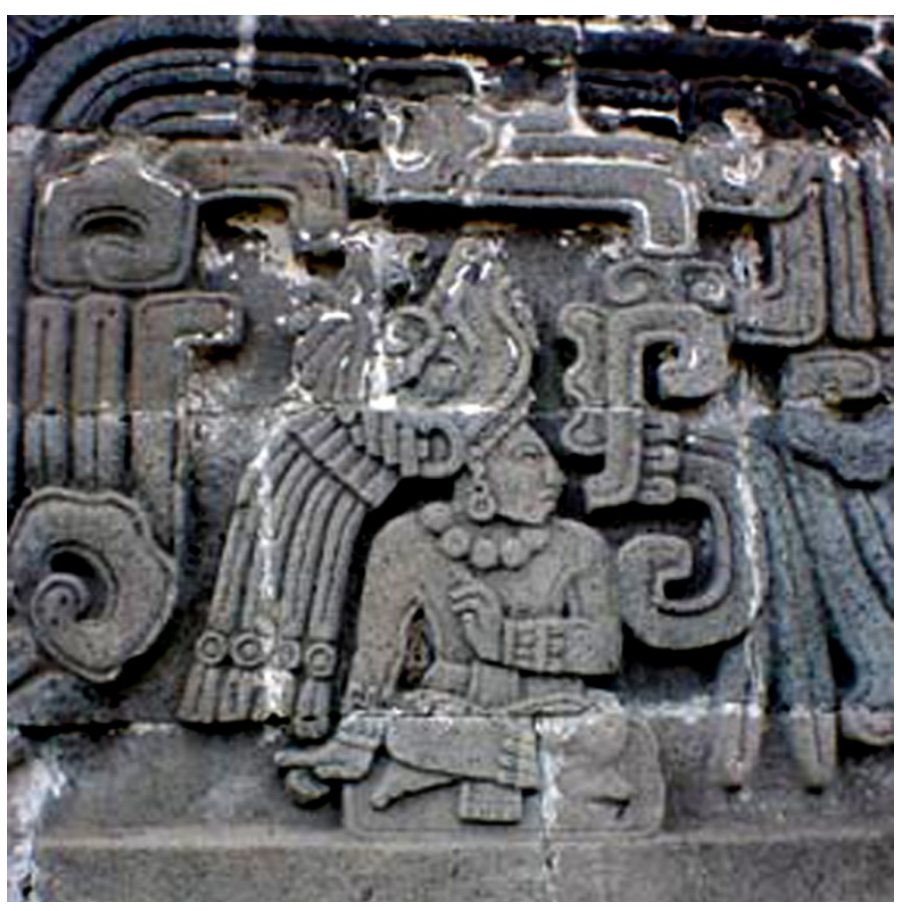

$\mathrm{B}$

Fotografía 2. A) Pirámide de las Serpientes Emplumadas, Xochicalco. B) Alto relieve con uno de los altos dignatarios reunidos en Xochicalco. Fotos: Juan Gabriel Ocampo.

El Juego de Pelota Oriente, por su parte, acompaña las estructuras dedicadas a los dioses y aunque no posee la fuerza del Juego de Pelota Norte, tiene una ubicación, con la cual, recibe para la Acrópolis al sol del naciente.

Cerca del Juego de Pelota Norte, se tiene y no de una manera fortuita, el ingreso a los sectores cavernosos y subterráneos, que llevan al observatorio astronómico. Evidencia ésta sobre la relación entre el inframundo y la existencia superior en un cielo que era leído e interpretado por los Xochicalcas hasta conseguir el conocimiento que les permitió conocer los secretos del tiempo (Ocampo, 2007).
Basado en los análisis del Juego de Pelota prehispánico mesoamericano, se podría concluir y recomendar:

El alto rendimiento a partir de la motivación del juego de pelota.

El juego de pelota implicaba, desde el punto de vista profano, la ganancia como juego de azar e, incluso, el posicionamiento social. Esta actividad, como tema del calmécac, demuestra la motivación que tiene el liderazgo del deportista exitoso.

En el ámbito sacro el juego de pelota significaba la fertilidad del pueblo, la divinidad para algunos y el poderío para el 

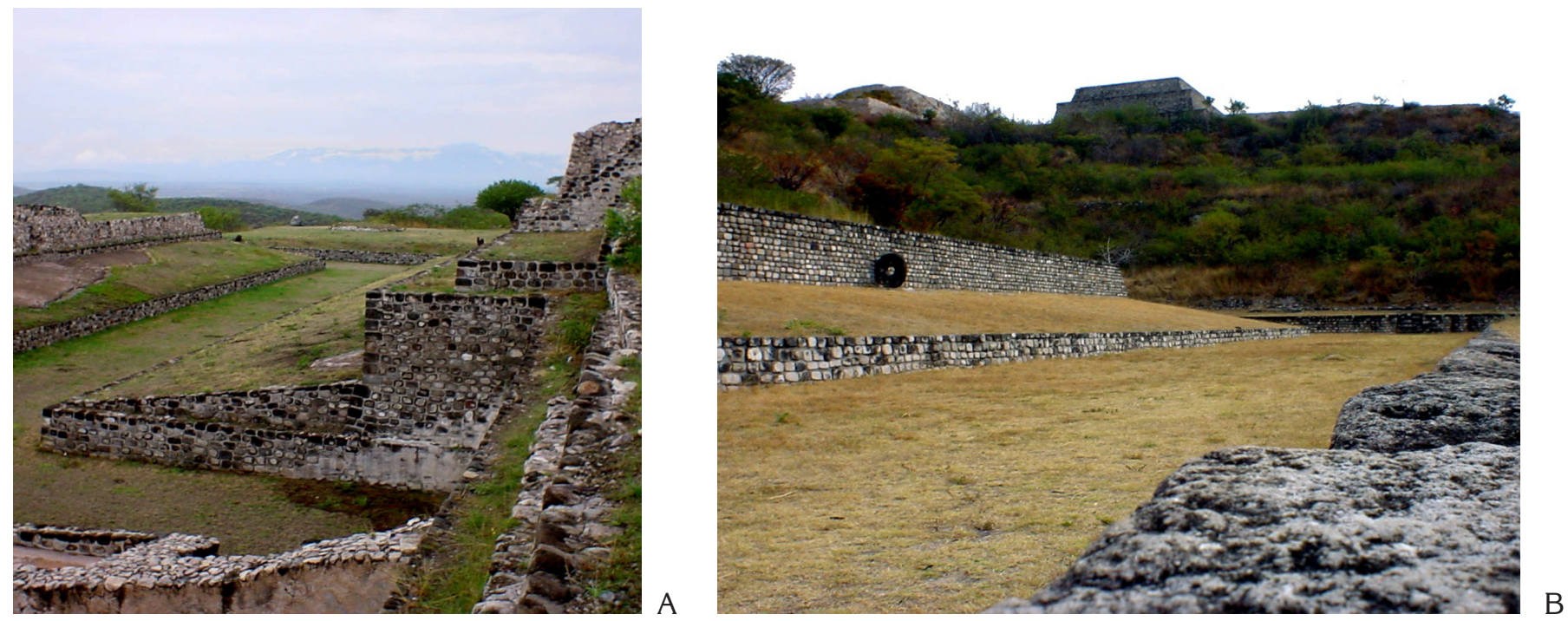

Fotografía 3. A) Juego de Pelota Oriente, Xochicalco. B) Juego de Pelota Sur, Xochicalco. Foto: Juan Gabriel Ocampo.
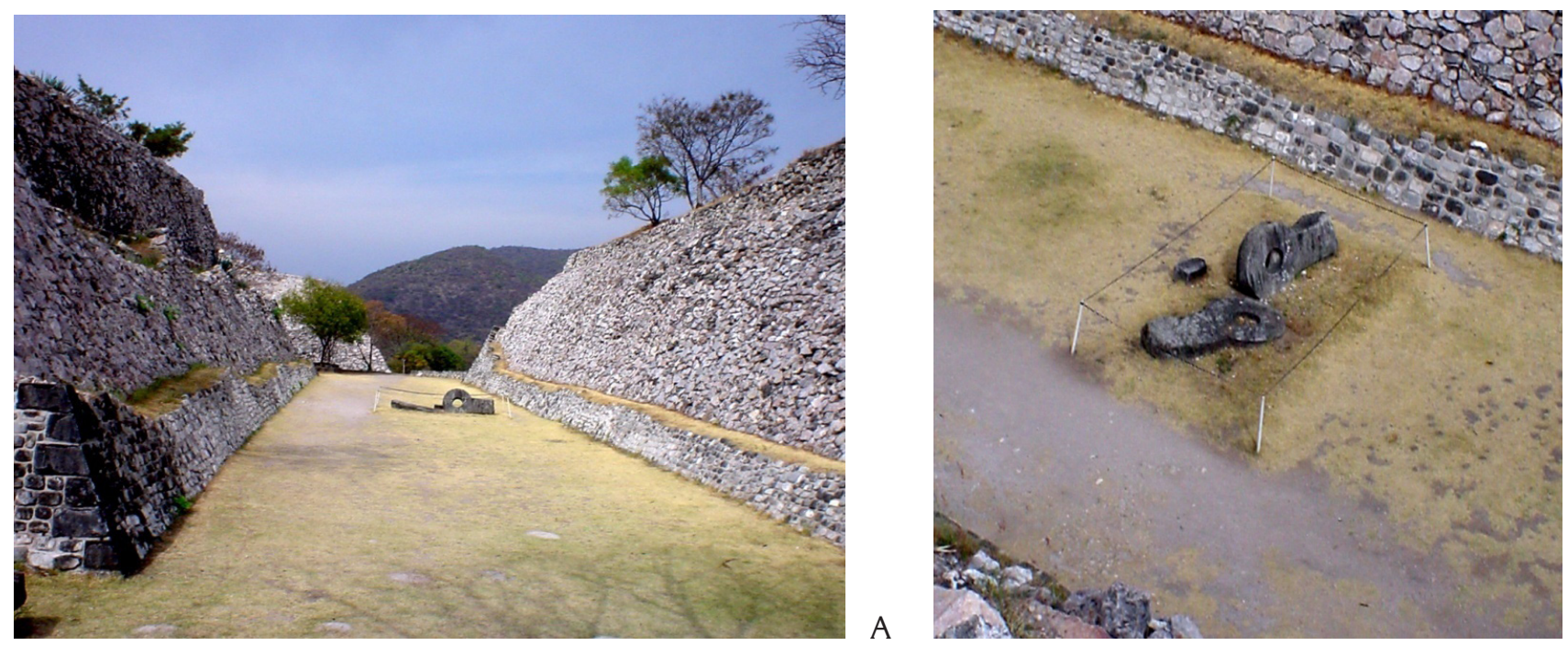

B

Fotografía 4. A) Juego de Pelota Norte, Xochicalco. B) Marcadores en Juego de Pelota Norte, Xochicalco. Foto: Juan Gabriel Ocampo.

Huey Tlatoani. Encontrar la muerte para alcanzar la divinidad implicaba una relación profunda entre la actividad física y la cosmovisión.

Propuesta categórica: Juegos olímpicos históricos.

La cancha del juego de pelota no se utiliza con su forma original. Para el impedimento de su uso, Tomas de Torquemada, primer inquisidor general del Tribunal del Santo Ofi- cio, dio las órdenes del caso. La transformación del juego, a lo largo del tiempo, alcanzó al presente con el juego de los ulama, en el estado de Sinaloa o con el juego de la pelota mixteca, en el estado de Oaxaca.

Si bien el juego de los ulama o el juego de la pelota mixteca no alcanzarían a tener las condiciones suficientes para llegar 
a la categoría de juegos olímpicos, dado su carácter regional, el juego de pelota prehispánico podría caber en dicha categoría. El inconveniente insalvable es el que tiene que ver con su inexistencia actual, debida a las órdenes del Tribunal de la Inquisición. Ahora bien, un deporte que tuvo presencia por más de 3.000 años y existió en una región que comprende a varios de los países existentes en la actualidad, bien vale la pena la presentación de una propuesta sobre la creación de una categoría de "Deportes Olímpicos Históricos" que, a pesar de no ser programados o existir en actividad, tienen un nivel de impacto significativo en la cultura de una región.

Agradecimientos: Financiación: Este estudio fue financiado por la Universidad Nacional de Colombia, en la investigación "Lógica y Arquitectura", mediante la convocatoria nacional de investigación y de creación artística 2010-2012 (HERMES 12579). Conflicto de intereses: El autor declara que no existe ningún conflicto de intereses que ponga en riesgo la validez de los resultados presentados.

\section{BIBLIOGRAFÍA}

1. Anónimo. 2003. Popol Vuh. Fondo de Cultura Económica (México).171p.

2. BRICEÑO JÁUREGUI, M. 1990. Los juegos olímpicos en la antigüedad. Instituto Caro y Cuervo. (Colombia) 219p.

3. FERNÁNDEZ, L.; NEGROE, G.; LACADENA, A.; YAM, M.; FERNÁNDEZ, S.; LÓPEZ, A. 2006. Historia Prehispánica y Colonial de Yucatán. Mc Graw Hill. (México). 195p.

4. MARTÍNEZ CADENA, M.I. 2007. El juego de pelota prehispánico: Un fenómeno de comunicación. Rev. D.A.D.U. (México). 2:101-112.

5. MARTÍNEZ CADENA, M.I. 2008. La arquitectura: Un sistema simbólico dentro de la totalidad de la cultura. Rev. D.A.D.U. 5:215-221.

6. NIELSEN REYES, F. 1977. El creador de los juegos olímpicos modernos, Barón Pierre de Coubertin: apostol de la hermandad. Universidad de Texas. (Estados Unidos). 105p.

7. NOGUERA, A.P. 2004. El reencantamiento del mundo. PNUMA. (Colombia). 206p.

8. OCAMPO HURTADO, J.G. 2007. Xochicalco, ciudad vertical prehispánica. Rev. D.A.D.U. 2:75-88.
9. RUIZ RESTREPO, C.E. 2010. Educación y humanismo en la vida universitaria. Ed. Página Maestra Editores (Colombia). 375p.

10. SAHAGÚN, B. de. 1829. Historia General de las Cosas de Nueva España. Imprenta del ciudadano Alejandro Valdés (México). 397p.

11. SCHELE, L.; MILLER, M.E. 1986. The Blood of Kings, Dynasty and Ritual in Maya Art. Kimbell Art Museum (Estados Unidos). 335p.

12. TALADOIRE, E. 2000. El juego de pelota mesoamericano. Origen y desarrollo. Rev. Arqueología. Mexicana. 8(44):20-27.

13. URIARTE CASTAÑEDA, M.T. 1991. El juego de pelota en Mesoamérica, raíces y supervivencia. Siglo XXI Editores. (México). 413p.

Recibido: Enero 11 de 2012

Aceptado: Marzo 21 de 2012 